\title{
Determination of synovial fluid hyaluronate concentration and polymerisation by high performance liquid chromatography
}

\author{
HERKKO SAARI AND YRJÖ T KONTTINEN \\ From the Fourth Department of Medicine, Helsinki University Central Hospital, Helsinki, Finland
}

SUMMARY High performance liquid chromatography (HPLC) with a size exclusion column and ultraviolet monitoring was used to study the effect of synovial fluid hyaluronate concentration and degree of polymerisation on viscosity and mucin clot formation. Rotational viscometry measurements showed an exponential relation between the synovial fluid hyaluronate concentration and relative viscosity, the viscosity increasing particularly steeply with hyaluronate concentrations exceeding $2-2.5 \mathrm{mg} / \mathrm{ml}$. The scattering of individual values observed around the expected curve was eliminated when both the hyaluronate concentration and its degree of polymerisation were taken into account. Hyaluronate concentration and degree of polymerisation also correlated with the quality of mucin clot, though only HPLC provided more detailed quantitative information about this association. Because HPLC allows reproducible and rapid simultaneous analysis of the synovial fluid hyaluronate concentration and the degree of polymerisation in small volumes of unprocessed samples it can be used in well equipped rheumatological units to replace other methods used previously.

The effects of the hyaluronate concentration and its molecular weight on synovial fluid viscosity and mucin clot formation have so far evaded exact characterisation because of the lack of a rapid and reproducible method suitable for the assessment of both hyaluronate concentration and molecular weight without pretreatment of samples. ${ }^{12} \mathrm{We}$ describe such a method based on high performance liquid chromatography (HPLC) in combination with a size exclusion column and ultraviolet monitoring. In our work we studied the effect of the concentration and degree of polymerisation of hyaluronate on viscosity and mucin clot formation in human synovial fluid obtained from meniscus and from patients with osteoarthritis and patients with various inflammatory joint diseases.

\section{Materials and methods}

SYNOVIAL FLUIDS

Synovial fluid was obtained from the knee joints of 21 patients: by arthrocentesis of the knee joints of 10

Accepted for publication 1 December 1988.

Correspondence to Dr H Saari, Fourth Department of Medicine, Helsinki University Central Hospital, Unioninkatu 38, SF-00170 Helsinki, Finland. patients with rheumatoid arthritis, ${ }^{3}$ of one patient with chronic Reiter's disease, ${ }^{4}$ and of four patients with acute reactive arthritis and by arthroscopy of the knee joints of six patients with lesion of the knee meniscus. The synovial fluid was aspirated by syringe and immediately transferred to sterile test tubes and centrifuged at $1000 \mathrm{~g}$ for 10 minutes to obtain the cells in pellet form. The synovial fluid samples were stored at $-20^{\circ} \mathrm{C}$ without preservatives. For HPLC $10 \mu \mathrm{l}$ of the synovial fluid sample was diluted to $2.0 \mathrm{ml}$ with $50 \mathrm{mM}$ sodium phosphate buffer $\mathrm{pH} 6.5$, and $50 \mu \mathrm{l}$ was then injected into the HPLC apparatus.

HIGH PERFORMANCE LIQUID

CH ROM A T O G R A P H Y

For exclusion chromatography a Waters associated chromatography pump (model 6000A with sample loop of $50 \mu \mathrm{l}$ ) was equipped with a Toyo Soda TSK 6000 PW exclusion column. Peak elutions were monitored with a Pye Unicam LC3 online ultraviolet detector (wavelength $206 \mathrm{~nm}$ ) with a Shimadzu Chromatopac C-R3A integrator. The elution buffer was $50 \mathrm{mM}$ sodium phosphate $\mathrm{pH} 6.5$ and the flow $1.0 \mathrm{ml} / \mathrm{min}$. All measurements were performed at room temperature. 
V I S C O I I Y

The viscosity of the synovial fluid was measured in a $2 \mathrm{ml}$ sample at $37^{\circ} \mathrm{C}$ by rotation viscometry with four different shear rates.

MUCIN CLOT

Acetic acid ( $4 \mathrm{ml}$ of $2 \%$ ) was added to $1 \mathrm{ml}$ synovial fluid. The mixture was vigorously shaken and allowed to stand at $4^{\circ} \mathrm{C}$ for 15 minutes. After mucin clot formation the samples were centrifuged at room temperature for 15 minutes at $5000 \mathrm{~g}$. The supernatant was carefully decanted and dialysed to remove acetate. Dialysis was performed with $100 \times$ volume $50 \mathrm{mM}$ sodium phosphate buffer $\mathrm{pH} 6.5$ as external medium. After four hours' dialysis the medium was changed and the dialysis continued overnight at $4^{\circ} \mathrm{C}$ with constant magnetic stirring. The viscous mucin precipitates were washed twice with distilled water and homogenised into the $50 \mathrm{mM}$ sodium phosphate buffer $\mathrm{pH} 6 \cdot 5$. The resolubilisation was performed with constant magnetic stirring at $4^{\circ} \mathrm{C}$ for 48 hours.

DEGRADATION OF HYALURONATE

The synovial fluid samples were treated with hyaluronidase as follows: 0.725 units of hyaluronidase were added to $1 \mathrm{ml} 1 / 40$ diluted synovial fluid and the mixture incubated for 12 hours at $37^{\circ} \mathrm{C}$.

CALIBRATION OF THE HPLC COLUMN

Hyaluronate standards for calibration of the HPLC column were kindly provided by Pharmacia Diagnostica AB (Uppsala, Sweden). To obtain appropriate molecular weight resolution, even with high molecular weight hyaluronate, it was necessary to use hyaluronate samples diluted to approximately $10 \mathrm{mg} / \mathrm{ml}$. Each molecular weight standard was chromatographed separately. The molecular weight determinations of the standards were performed by the manufacturer by low angle laser light scattering.

\section{RADIOMETRIC DETECTION OF}

HYA L URONATE

The hyaluronate 50 test, which is based on the use of specific hyaluronate binding protein isolated from bovine cartilage, was used for the radiometric detection of hyaluronate from synovial fluid samples. ${ }^{5}$ A 2000 to 10000 -fold dilution of the synovial fluid sample was usually required for the test. Radioactivity was measured by an LKB 1975 RiaGamma automatic gammacounter. Each determination was performed in duplicate for both standards and samples. Hyaluronate test 50 kits were obtained from Pharmacia Diagnostica AB (S 75182, Uppsala, Sweden).

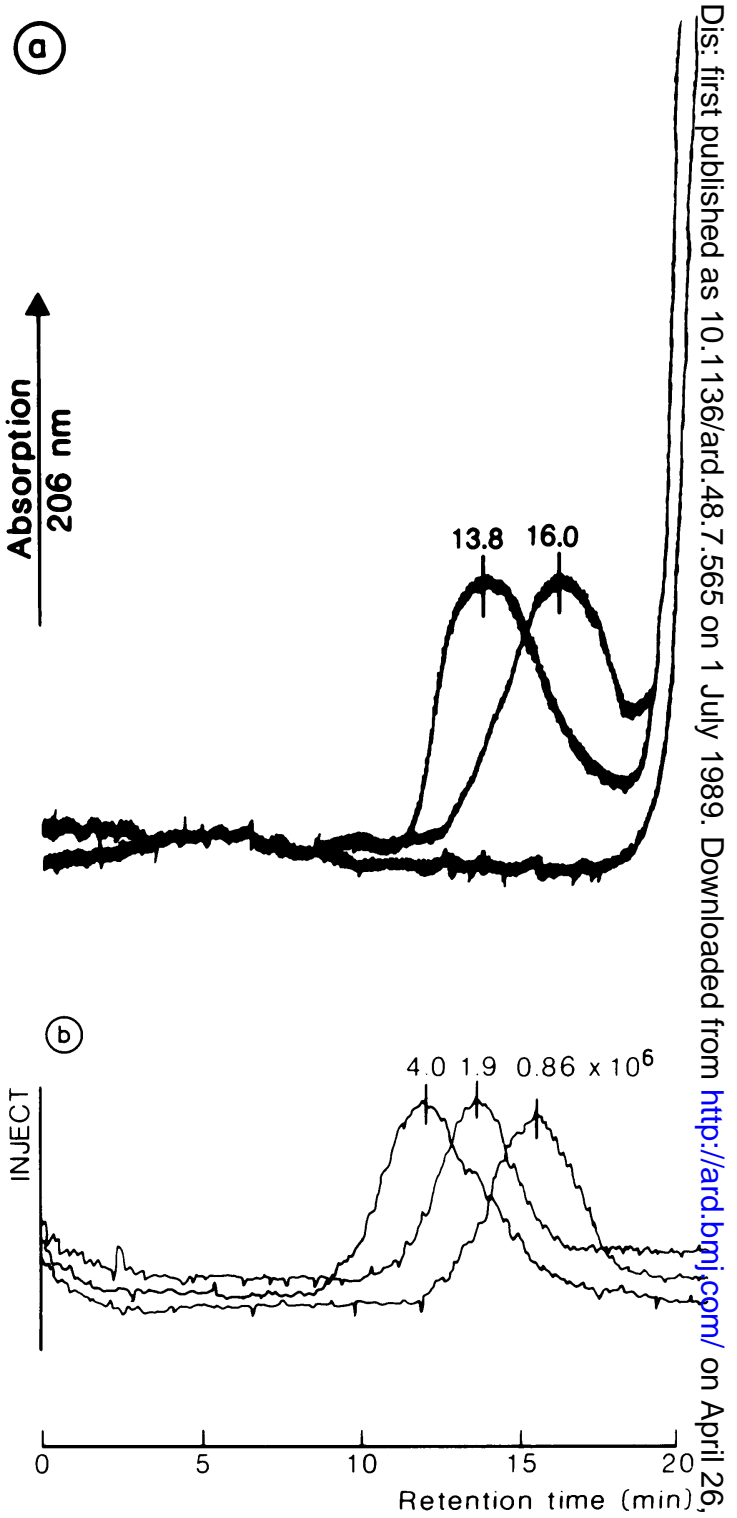

Fig. 1 (a) High performance liquid chromatography (HPLC) chromatograms of two synovial fluid samples having different degrees of polymerisation of hyaluronate. The hyaluronate peak at retention time 13.8 minutes represents a high viscosity, non-inflamed synovial fluid sample, and the peak at 16.0 minutes shows the chromatographic finding of a typical inflamed synovial fluid sample. The hyaluronate content of both synovial flui samples was close to $2 \mathrm{mg} / \mathrm{ml}$. The lower curve shows the hyaluronidase susceptibility of these peaks. (b) HPLC chromatograms of three different hyaluronate standards with molecular weights of $4.0 \times 10^{6}, 1.9 \times 10^{6}$, and $0.86 \times 1$ and retention times of $13.62,14.68$, and 15.53 minutes respectively. 
S T A T I S T I C S

The relation between two variables was studied by linear regression analysis.

\section{Results}

VISCOSITY AND SYNOVIAL FLUID

HYA L URONATE

Comparison of paired synovial fluid samples with the same concentration $(2 \mathrm{mg} / \mathrm{ml}$, peak heights; Fig. 1a) but different molecular weights (retention time; Fig. 1a (for molecular weight calibration, see Figs $1 \mathrm{~b}$ and 2)) clearly disclosed the dependency of relative viscosity on the degree of polymerisation of synovial fluid hyaluronate (Fig. 3). In these experiments the identification of hyaluronate was based on its sensitivity to hyaluronidase treatment. In addition, a specific radiometric ${ }^{125}$ I hyaluronate binding protein test and fraction collection from HPLC elution solution showed that no low molecular hyaluronate was masked behind the protein peak. Figure 1b shows somes results of the molecular weight calibration of the HPLC column. The molecular weights of the hyaluronate in the synovial fluid samples were obtained from the calibration curve (Fig. 2). It should be noted that molecular weight corresponds logarithmically to retention time and that compounds with great molecular differences elute in a very narrow retention time range.

In another attempt to study the effect of the concentration and molecular weight of synovial fluid hyaluronate on viscosity the relative viscosities of 22

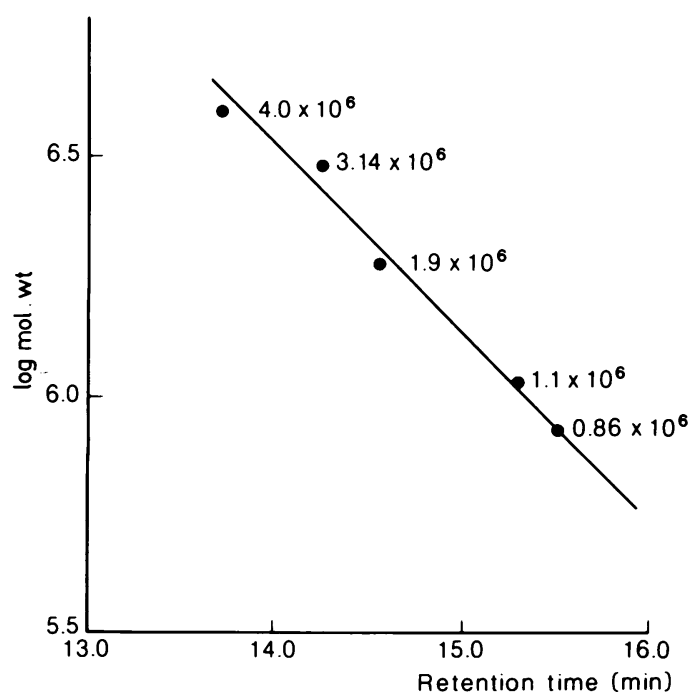

Fig. 2 Calibration curve of the high performance liquid chromatography column.

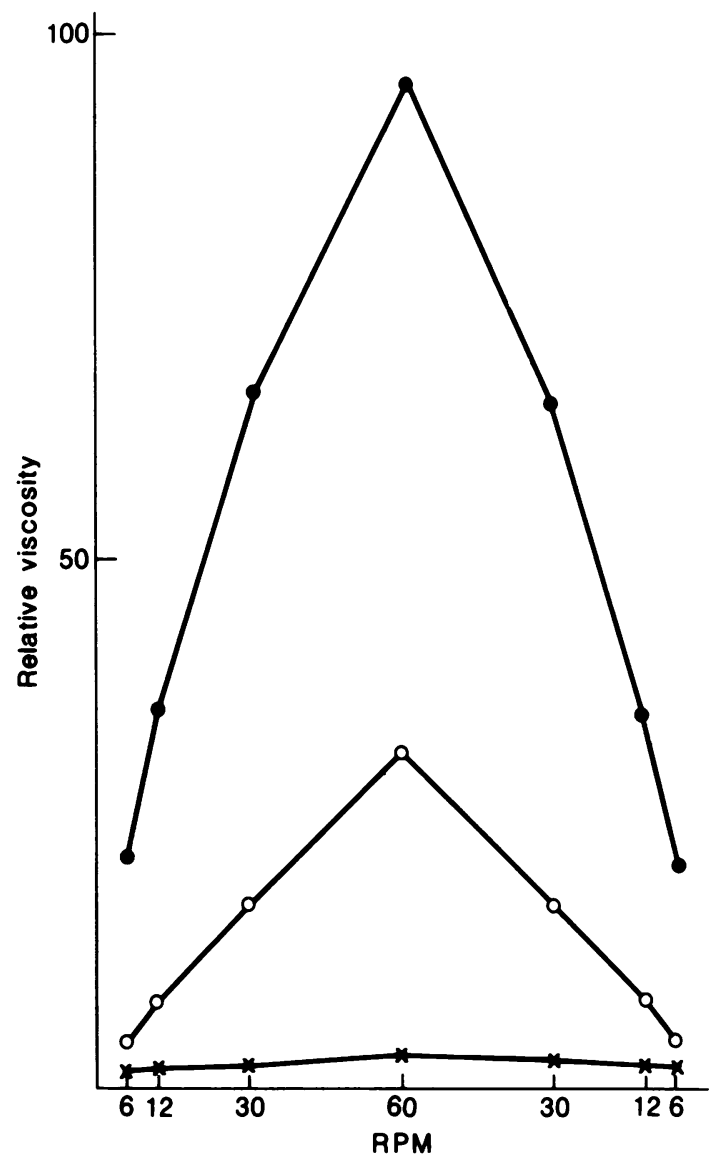

Fig. 3 Dependence of viscosity on the degree of polymerisation of hyaluronate in synovial fluid. Relative viscosity on the $y$ axis and shear rates on the $x$ axis. The upper curve shows the effect of highly polymerised hyaluronate in synovial fluid (retention time $13 \cdot 8$ minutes) and the middle trace hyaluronate in a less polymerised form (retention time 16.0 minutes). The lowest curve shows the effect of hyaluronidase treatment.

synovial fluid samples from patients with different inflammatory joint diseases were first measured against the hyaluronate concentration, determined by HPLC (Fig. 4a). There was an exponential relation between viscosity and hyaluronate concentration, but the scattering of individual values around the exponential curve was quite large (Fig. 4a). It was also observed that the differences in relative viscosities in samples with the same hyaluronate concentration were associated with differences in the degree of polymerisation of hyaluronate (see, for example, the experiments depicted in Figs 1 and 3). Furthermore, if a high viscosity sample was 
serially diluted and the viscosity plotted against the hyaluronate concentration measured by HPLC there was only insignificant scattering between the observed and expected values (Fig. 4b). These experiments show that the relative viscosity of synovial fluid is a function of both concentration and degree of polymerisation.

MUCIN CLOT AND SYNOVIAL FLUID

HY A L U RON A T E

It was shown that almost all of the synovial fluid hyaluronate was present in the presolubilised mucin precipitate; no hyaluronate measurable by HPLC was found in the supernatant (Fig. 5) The ${ }^{125} \mathrm{I}$ hyaluronate binding protein radiometric test confirmed that only minor amounts of non-precipitated hyaluronate were in the mucin precipitate supernatant when the mucins were precipitated according to the method described elsewhere ${ }^{6}$ (Table 1). Furthermore, comparisons of the retention times of hyaluronate in the untreated synovial fluid and in the presolubilised mucin clot disclosed that no marked degradation occurred during precipitation and resolubilisation. The solubilisation of the mucin clot was not complete, however, as insoluble material still remained in the test tube after 48 hours of stirring at $4^{\circ} \mathrm{C}$. Even treatment of this material with $4 \mathrm{M}$ guanidinium hydrochloride had no effect. In contrast with synovial fluid hyaluronate, only a
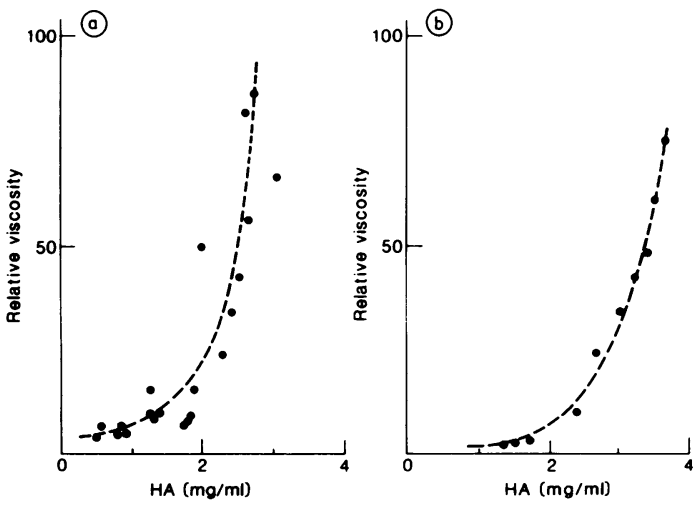

Fig. 4 (a) Correlation between viscosity and hyaluronate concentration in 22 synovial fluid samples. The y axis shows the relative viscosity, the $x$ axis the hyaluronate concentration as determined by high performance liquid chromatography. An exponential curve was obtained. (b) One synovial fluid sample, with a high relative viscosity and hyaluronate concentration $3.9 \mathrm{mg} / \mathrm{ml}$ was diluted with $0.9 \%$ saline, and the viscosity and hyaluronate concentration were determined after each dilution.
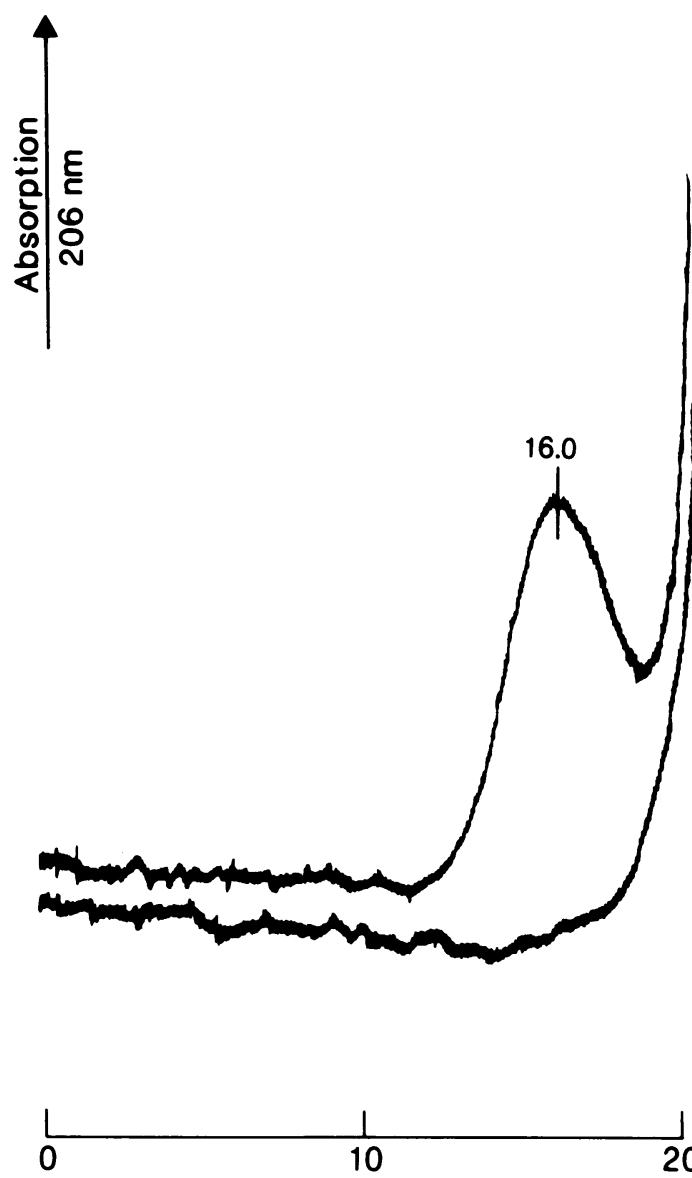

Retention time ( $\mathrm{min})$

Fig. 5 The upper trace represents a high performance liquid chromatography (HPLC) chromatogram of a typicaß 'good' mucin clot precipitate after resolubilisation in $50 \mathrm{mM}$ sodium phosphate pH 6.5. The lower trace shows the corresponding supernatant, in which no hyaluronate was detected by HPLC.

minor fraction of total synovial fluid protein copres cipitated with the synovial fluid hyaluronate, most of the protein accordingly being found in the muciu clot supernatant (Table 1). There was no significare correlation between viscosity and synovial fluie protein $(r=-0.265, p>0.05)$. When estimated by the criteria of Cohen, ${ }^{6}$ the quality of mucin clots (poor, moderate, good) correlated well with th hyaluronate concentration in the synovial fluid samples (Table 1). The good mucin precipitate samples also had a high relative viscosity and a high degree of polymerisation (Table 1). 


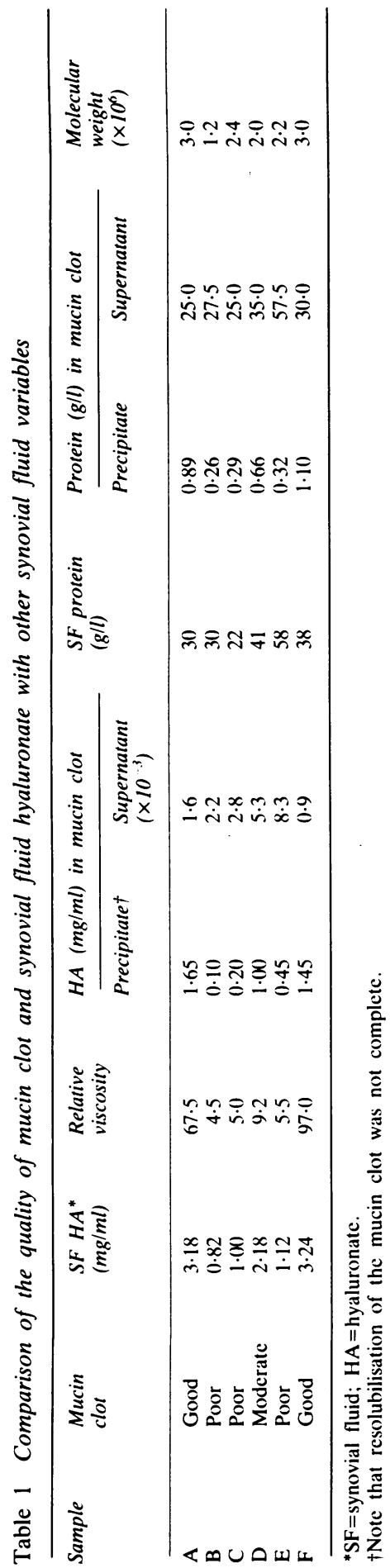

\section{Discussion}

Some fundamental propertits of synovial fluidnamely, viscosity and mucin clot formation, depend on the concentration and degree of polymerisation of the synovial fluid hyaluronate. ${ }^{6-10}$ These can be studied in small samples simultaneously by rapid and sensitive HPLC; pooled samples are not necessary. The viscosity of individual synovial fluid samples was shown to be a function of both concentration and degree of polymerisation. Owing to the exponential relation between the hyaluronate concentration and the relative viscosity, the rise in viscosity was particularly steep when the concentration exceeded $2-2.5 \mathrm{mg} / \mathrm{ml}$.

The effect of molecular weight on viscosity was evident in studies of paired samples with the same hyaluronate concentration but different molecular weights, in samples obtained from patients with various joint diseases and in dilution studies of individual samples. The degree of polymerisation of hyaluronate has previously been studied by conventional gel chromatography ${ }^{12}$ in dialysed samples adjusted to a suitable density by the addition of caesium chloride and ultracentrifuged for two days. The present technique uses unprocessed samples, and one run takes no longer than 15-20 minutes, depending on the retention time. This diminishes the risk of artefact depolymerisation during sample processing and analysis. Our study shows quantitatively the way in which the degree of polymerisation of hyaluronate and its concentration determine its rheological properties. Thus an extension of older pioneering work on synovial fluid hyaluronate has been made possible by modern biochemistry. Our observations also explain why viscosity is less reliable than previously thought in the classification of effusions.

Quantitative studies of the time honoured mucin clot precipitation test showed that almost all synovial fluid hyaluronate is found in the mucin clot, whereas only minor amounts of synovial fluid protein coprecipitate with the hyaluronate, though these may have important functional effects. ${ }^{11}$ HPLC anlaysis disclosed that there was no change in the molecular weight of hyaluronate during precipitation/resolubilisation. Our study confirms an earlier observation that there is a correlation between the quality of mucin clot and the synovial fluid hyaluronate concentration and its degree of polymerisation, ${ }^{8}$ albeit only a rough correlation. When the advantages of synovial fluid hyaluronate HPLC analysis are considered it is probable that in well equipped rheumatological units this method will replace mucin clot and ultracentrifugation and gel filtration in hyaluronate research. 


\section{Saari, Konttinen}

This study was financially supported by the Sigrid Juselius Foundation, Koe-eläinten suojelun säätiö, Reumatautien tutkimussäätiö, and Duodecim. Hyaluronate molecular weight standards were kindly provided by Pharmacia Diagnostica AB, who are gratefully acknowledged. Yrjö T Konttinen is senior investigator of the Arthritis Foundation in Finland.

\section{References}

1 Scher I, Hamerman D. Isolation of human synovial-fluid hyaluronate by density-gradient ultracentrifugation and evaluation of its protein content. Biochem J 1972; 126: 1073-80.

2 Bjelle A, Andersson T, Granath K. Molecular weight distribution of hyaluronic acid of human synovial fluid in rheumatic diseases. Scand J Rheumatol 1983; 12: 133-8.

3 Arnett F C, Edworthy S M, Bloch D A, et al. The American Rheumatism Association 1987 revised criteria for the classification of rheumatoid arthritis. Arthritis Rheum 1988; 31: 315-24.

4 Calin A. Reiter's syndrome. In: Kelley W N, Harris E D Jr, Ruddy S, Sledge C B, eds. Textbook of rheumatology. 2nd ed. Philadelphia: Saunders, 1985: 1007-20.
5 Brandt R, Hedlöf E, Åsman I, Bucht A, Tengblad A. $\mathbb{~}$ convenient radiometric assay for hyaluronan. Acta Otolaryngot [Suppl] [Stockh] 1987; 442: 31-5.

6 Cohen A S, Goldenberg D. Synovial fluid. In: Cohen A S, e⿻ Laboratory diagnostic procedures in the rheumatic diseases. 38 ed. Orlando: Grune and Stratton, 1985: 1-54.

7 Meyer K. Smyth E M, Dawson M H. The isolation of $\overline{\overline{c a}}$ mucopolysaccharide from synovial fluid. J Biol Chem 1939; 12 319-27.

8 Bollet A J. The intrinsic viscosity of synovial fluid hyalurongs acid. J Lab Clin Med 1956; 48: 721-8.

9 Cleland R L, Wang J L. Ionic polysaccharides. III. Dilut solution properties of hyaluronic acid fractions. Biopolymers 1970; 9: 799-810.

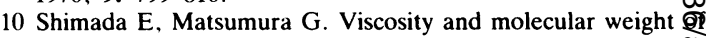
hyaluronic acids. J Biochem 1975; 78: 513-7.

11 Hutadilok N, Ghosh P. Brooks P M. Binding of haptoglobio inter- $\alpha$-trypsin inhibitor, and $\alpha_{1}$ proteinase inhibitor to synovit fluid hyaluronate and the influence of these proteins on its degradation by oxygen derived free radicals. Ann Rheum Dig 1988: 47: $377-85$. 